65 years was 2.9 (95\% CI: 1.6-5.2) and 5.8 (2.5-9.7) for males and females, respectively. After adjustment for the Gini coefficient, the RII increased to $4.2(2.6-6.6)$ and 6.3 (3.910.1) for males and females, respectively. Inequalities in treatment among older hypertensive females were similar in magnitude before and after adjustment for the Gini coefficient, with an estimated RII of $0.6(0.5-0.8)$ and $0.7 \quad(0.5-0.8)$ respectively.

Conclusion Multilevel analyses are required to better estimate inequalities. Chile currently needs interventions to improve the management of hypertension and simultaneously, decrease inequalities, first, in hypertension prevalence among younger males and females and, secondly, in its management among older hypertensive females.

The Chilean National Health Surveys 2003-2010-2017 were funded by the Chilean Ministry of Health.

This study is part of Alvaro Passi-Solar's PhD research, funded by the Chilean Ministry of Education (ANID-Conicyt).

\section{P34 SOCIOECONOMIC DISPARITIES IN THE DEVELOPMENT OF MULTIMORBIDITY IN SCOTLAND: THE BENEFITS OF APPLYING A LIFE COURSE LONGITUDINAL APPROACH}

${ }^{1} \mathrm{G}$ Cezard*, ${ }^{1} \mathrm{~K}$ Keenan, ${ }^{2} \mathrm{~F}$ Sullivan. 'School of Geography and Sustainable Development, University of St Andrews, St Andrews, UK; ${ }^{2}$ School of Medicine, University of St Andrews, St Andrews, UK

\subsection{6/jech-2020-SSMabstracts. 128}

Background There is widespread acknowledgement that we need to go beyond the single disease framework when trying to understand health inequalities across the life course. In Scotland, evidence based on primary care records showed that more than half of the people aged 65 years and older had at least two chronic diseases. Multimorbidity, defined as the cooccurrence of two or more chronic diseases, was also shown to be more prevalent in women and those with lower socioeconomic status (SES). The vast majority of multimorbidity research (and the indicators used) is situated within a crosssectional framework, and a typical goal is to identify disease clusters at a single point in time.

We aim to investigate the social patterning of multimorbidity trajectories in Scotland using both cross-sectional and longitudinal approaches.

Methods We use the Scottish Longitudinal Study (SLS) which links 1991, 2001 and 2011 censuses, vital events and other administrative or publicly available data sets for a 5\% representative sample of the Scottish population. Our subsample focuses on 120000 SLS individuals aged 40 years and over at the time of the Scottish census 2001, linked to their Scottish census 2011, hospitalisation and death records. Our sample is followed for up to 10 years (next census), censoring for death or emigration. Multimorbidity is identified using hospitalisation data and the list of comorbidities of the Charlson Index using Quan et al. algorithm (2005). Educational level in 2001 and the Scottish Index of Multiple Deprivation (SIMD) are used as SES proxies. Poisson and Cox regressions are used to explore SES inequalities in multimorbidity index at baseline and over time.

Results As expected, we find that multimorbidity is socially patterned: Individuals with lower education and who live in more deprived areas experienced faster levels of multimorbidity accumulation. In a separate analysis, we use single-channel sequence analysis to characterise multimorbidity trajectories and sequences of disease onset and relate this to social inequalities.

Conclusion This study highlights the need to go beyond a cross-sectional approach in researching multimorbidity differences. It shows the influence of the timing of and sequencing of disease onset in shaping social health inequalities in later life and it also provides a characterisation of multimorbidity progression through the application of longitudinal methods used in life course studies. With new data linkages, capturing detailed disease onset, and timing linked with social factors, research of the social patterning of multimorbidity progression can be advanced and fed into prevention strategies.

\section{P35 AN INDIVIDUAL PARTICIPANT DATA META-ANALYSIS EXAMINING HEALTH INEQUALITIES FACING OLDER LESBIAN, GAY AND BISEXUAL PEOPLE AGED 50 AND OVER IN THE UNITED KINGDOM}

D Kneale*. EPPI-Centre, University College London, London, UK

\subsection{6/jech-2020-SSMabstracts.129}

Background Older lesbian, gay and bisexual (LGB) people were born at a time same sex activity between men was a criminal offence, and where social and legislative conditions permitted discrimination against sexual minorities across a spectrum of domains. Minority Stress Theory posits that LGBT people are at an elevated risk for poorer health because of their exposure to social stress related to prejudice and stigma. Modelling the health and care trajectories of lesbian, gay and bisexual (LGB) is essential to identify inequalities and support needs, yet because of the small sample of LGB people in any one survey, current evidence relies on studies that have poor generalisability and low power. This study assesses the magnitude of health and care inequalities among older LGB people across ten outcomes, informed by evidence on the health trajectories and distinct history of LGB people in the UK.

Methods A systematic review was conducted of representative data sources on older LGB and heterosexual people's health and care status in the UK. Individual Participant Data (IPD) meta-analysis was employed to synthesise data. To account for the intricacies of individual datasets, the analysis employed a two-stage approach where an odds ratio (OR) and standard error was calculated for each dataset individually, before being meta-analysed through DerSimonian and Laird random effects models.

Results Our largest model incorporated data from 25 different datasets and provided an unparalleled sample size (over 2,500 LGB men and women) to measure the magnitude of sexualitybased health inequalities in later life. We find that among men aged 50 and over, being gay, bisexual or having another nonheterosexual orientation is associated with an increased risk of reporting a long-term illness and limitations due to health or illness. Indicators of mental health also suggest that gay and bisexual men are more likely to report low life satisfaction and substantially more likely to have attempted suicide over their life time (Adjusted Odds Ratio: 2.29; 95\% Confidence Interval: 1.19-4.42). We find that among women, differences in health are apparent with regards to self-rated health as well as with engagement with risky health behaviours with higher levels of smoking and frequent alcohol consumption. 
Conclusion The findings corroborate the minority stress theory, but they also generate new questions for researchers around when and why these inequalities emerge.

\section{P36 REGIONAL AND SOCIOECONOMIC DISPARITIES IN CHILD-TO-ADOLESCENT GROWTH TRAJECTORIES ACROSS GENERATIONS IN CHINA}

M Gao*, J Wells, L Li. GOS Institute of Child Health, University College London, London, UK

10.1136/jech-2020-SSMabstracts. 130

Background China has experienced rapid economic development since the 1990s, which has led to regional development inequality and could have an impact on the physical growth in Chinese children. Study about changes in growth trajectories across generations by region and socioeconomic (SES) factors is needed for informing policy to reduce inequality.

Methods We used the longitudinal data of 5118 children from China Health and Nutrition Survey (CHNS), who were born during 1981-2000 and whose height and weight were measured between 7-18y during 1991-2015. Four birth cohorts were derived (1981-85, 86-90, 91-95 and 96-2000) and mixed-effects fractional polynomial functions were applied to estimate child-to-adolescent growth trajectories by gender and cohort. Growth trajectories were further stratified by region (west, central, east and northeast) and urban/rural separately. Finally, both geographic and parental SES determinants (loghousehold income, paternal occupation and maternal education) were adjusted to estimate their association with childhood physical growth across cohorts.

Results Mean growth trajectories for height and BMI both shifted upwards across cohorts. The increase in BMI between the oldest (1981-85) and youngest (1996-2000) cohorts was $\sim 0.8 \mathrm{~kg} / \mathrm{m}^{2}$ at $9 \mathrm{y}$ (both genders) and remained in boys while narrowed in girls since late adolescence $\left(0.4 \mathrm{~kg} / \mathrm{m}^{2}\right.$ at $\left.17 \mathrm{y}\right)$. The increase in height widened pre-puberty $(5.7 \mathrm{~cm}$ in boys and $4.4 \mathrm{~cm}$ in girls at $13 \mathrm{y}$ ) and decreased thereafter. There were evident regional disparities in growth: gender-/cohort-specific BMI trajectories for children from the east region lay above those from the northeast region, followed by those for children from central/west areas. Height trajectory from northeast was the highest, followed by east, central and west regions. Growth increment across cohorts showed a similar regional pattern (e.g. children in west experienced the smallest growth increment across cohorts).

Urban children had higher BMI, were taller and had greater BMI increment while smaller height increment across cohorts than their rural counterparts, thus the urban-rural difference widened in BMI while narrowed in height across generations. Higher parental SES was associated with higher BMI and taller stature. The strength of relationship between maternal education with children's height was stronger in younger than in older cohorts.

Conclusion While children from urban and east region had higher BMI and greater BMI increment across cohorts, children from underdeveloped western China remain to be the shortest and should be maximized their potential of linear growth. The positive relationship between parental SES and children's BMI, which was opposite to the evidence in Western countries, may reflect a different mechanism in developing countries.

\section{P37 IDENTIFYING LONG-TERM, HIGH-DOSE USERS OF OPIOID DRUGS PRESCRIBED FOR CHRONIC NON- CANCER PAIN IN THE COMMUNITY}

1J Bailey*, ${ }^{2} S$ Gill, 1,2R Poole. 'School of Health Sciences, Bangor University, Bangor, UK;

${ }^{2}$ Betsi Cadwaladr University Health Board, NHS Wales, Wrexham, UK

\subsection{6/jech-2020-SSMabstracts. 131}

Background Studies of prescribing of opioid medication for chronic pain in the UK population show that it has increased sharply over the past 25 years. Although overall rates may have begun to decline recently, longer-term use and prescribing of 'strong' opioids continue to rise. Whilst analysis of data at a population level can tell us much about the use of these medications, an analysis at an individual level is needed to understand which patients, and how many, are at greatest risk from these potentially harmful drugs. Harms associated with these drugs are related to dose and length of use. The aim of this study was to develop a method to identify patients prescribed long-term, high doses of these drugs in the community and to assess the prevalence of such use.

Methods Details of all opioid prescriptions issued over a fourmonth period were collected from two demographically dissimilar GP practices. A total of 22,841 patients were registered at the practices, $1488(6.5 \%)$ of whom were prescribed opioids in the study period. Exhaustive examination of prescription data identified all patients who were prescribed oral morphine equivalent (ME) doses of $120 \mathrm{mg}$ /day or more in the census period. An examination of the prescription histories of these patients indicated those who had been prescribed opioids at this level for a year or more.

Results Every patient who met our criterion of $\geq 120 \mathrm{mg} / \mathrm{day}$ ME for a year or more was being prescribed that level as a single drug of morphine, oxycodone or fentanyl, irrespective of opioid polypharmacy. Across the two practices, 1.71/1000 patients were identified as long-term, high-dose users of opioid medication for chronic non-cancer pain. Prevalence was similar in the two practices. Unadjusted extrapolation suggests that there are over 100,000 similar patients in the UK.

Conclusion This study provides a simple, reliable and practical means of identifying patients prescribed long-term high-dose opioid medication for chronic pain. These regimens are unlikely to provide additional pain relief but increase the risk of harm to patients. They have marked negative effects on day-to-day functioning and quality of life. Patients of interest can be identified simply through their use of three drugs as threshold doses and above. This can help in the further investigation of dysfunctional medication use; in establishing national and local prevalence; in monitoring service provision; and in identifying associated factors, such as social deprivation and regional variation.

\section{P38 EXPLORING THE EFFECT OF NATIONAL INSTITUTE OF HEALTH AND CLINICAL EXCELLENCE GUIDELINES ON PRESCRIBING BEHAVIOUR FOR CHILDHOOD ATOPIC ECZEMA IN PRIMARY CARE WITH AN INTERRUPTED TIME SERIES}

${ }^{1}$ LIW Schreuders*, ${ }^{2} \mathrm{SJ}$ Ersser, ${ }^{3} \mathrm{C}$ Thompson. 'Work and Employment Relations Division, Leeds University Business School, Leeds, UK; ${ }^{2}$ Faculty of Health and Social Science, Bournemouth University, Dorset, UK; ${ }^{3}$ School of Healthcare, University of Leeds, Leeds, UK

10.1136/jech-2020-SSMabstracts. 132 